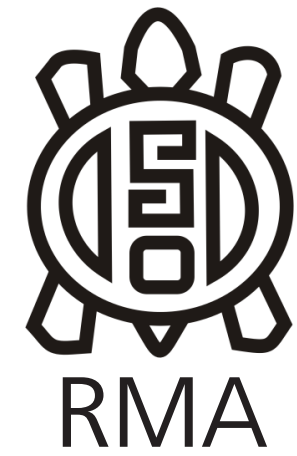

Arqueología

\title{
Obsidiana Varvarco: una nueva fuente en el noroeste de Patagonia (Neuquén, Argentina)
}

\author{
Varvarco obsidian: a new source in northwestern Patagonia (Neuquén, \\ Argentina)
}

\author{
María Victoria Fernández*, Pablo Leal**, Claudia Della Negra***, \\ Catherine Klesner ${ }^{* * *}$, Brandi L. MacDonald ${ }^{* * * * *}$, \\ Michael D. Glascock $k^{* * * *}$ y Ramiro Barberena ${ }^{* * * * * *}$
}

\begin{abstract}
${ }^{*}$ CONICET, Instituto de Investigaciones en Diversidad Cultural y Procesos de Cambio, Universidad Nacional de Río Negro, Argentina. E-mail: fernandezvicky5@gmail.com ${ }^{* *}$ CONICET, Instituto de Geociencias Básicas, Aplicadas y Ambientales de Buenos Aires, Facultad de Ciencias Exactas y Naturales, Universidad de Buenos Aires, Argentina. E-mail: prleal2000@gmail.com.

***Dirección General de Patrimonio Cultural, Provincia del Neuquén, Argentina. E-mail: patrimonioculturalprovincial@gmail.com

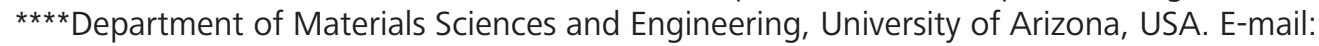
klesnerc@email.arizona.edu ${ }^{* * * * *}$ Archaeometry Laboratory, University of Missouri Research Reactor Center, Columbia, MO 65211. USA. E-mail: macdonbl@gmail.com ${ }^{* * * * *}$ Archaeometry Laboratory, University of Missouri Research Reactor Center, Columbia, MO 65211. USA. E-mail: GlascockM@missouri.edu ******Instituto Interdisciplinario de Ciencias Básicas (ICB), CONICET. Laboratorio de Paleoecología Humana, Facultad de Ciencias Exactas y Naturales, Universidad Nacional de Cuyo, Mendoza. UNCUYO-FCEN. E-mail: ramidus28@gmail.com
\end{abstract}

\section{Resumen}

En el norte neuquino existen depósitos piroclásticos que contienen nódulos de obsidianas. Las investigaciones en la región dieron a conocer las principales fuentes utilizadas por las poblaciones que allí habitaron. Para profundizar el conocimiento de cada fuente de obsidiana se adoptó un programa de estudio geoarqueológico y geoquímico. Así, se han descubierto nuevas ocurrencias de obsidianas, la más destacable de las cuales es la obsidiana Varvarco que aqui se presenta. Se dan a conocer los muestreos realizados en el valle del río Varvarco, su contexto geológico y geomorfológico, la presencia de obsidiana, el tipo de yacimiento, forma de presentación, distribución y abundancia. Luego, se describen las principales características observadas bajo microscopio petrográfico. Por último, se presentan los resultados de análisis geoquímicos de fluorescencia de rayos $X$ y activación neutrónica. A partir de los primeros la obsidiana Varvarco se diferencia de la de Cerro Huenul, pero no de la obsidiana Maule 1 y 2. Los segundos análisis discriminan bien esta nueva obsidiana de las ya conocidas para Neuquén. Los resultados obtenidos permiten reinterpretar la disponibilidad de recursos líticos de buena calidad para la talla y revisar las interpretaciones de movilidad e interacción de poblaciones en esta región.

Palabras Clave: Obsidiana; Varvarco; Geoquímica; Neuquén.

\begin{abstract}
In northern Neuquén there are pyroclastic deposits containing obsidian nodules. The researches in the region revealed the main sources used by the people that lived there. A program of geoarchaeological and geochemical study was adopted to extend the knowledge of each source of obsidian. Thus, new occurrences of obsidian have been discovered, the most notable of which is the Varvarco obsidian presented here. We present the samples points conducted in the valley of Varvarco river, its geological and geomorphological context, the presence of obsidian, the type of deposit, presentation form, distribution and abundance. Then, we described the main characteristics observed under a petrographic microscope. Finally, we present the results of $X$-ray fluorescence and neutron activation geochemical analysis. The Varvarco obsidian differs from that of Cerro Huenul, but not from the obsidian Maule 1 and 2 from the XRF. NAA discriminates well this new obsidian from those already known in Neuquén. The results obtained allow to reinterpret the availability of good quality lithic resources and to revise the interpretations of mobility and interaction of populations in this region.
\end{abstract}

Keywords: Obsidian; Varvarco; Geochemistry; Neuquén. 
La subducción de corteza oceánica por debajo del margen occidental del continente sudamericano generó varias etapas de intenso volcanismo de arco o retroarco, provocando la extrusión de importantes volúmenes de lava y subproductos volcánicos, entre ellos extensos depósitos piroclásticos que contienen nódulos de obsidianas. Sobre estos depósitos han actuado, desde entonces, múltiples procesos exógenos (González Díaz, Fauqué, Giaccardi y Costa, 2000; González Díaz, Giaccardi y Costa, 2003) que han erodado, transportado y/o redistribuido las obsidianas. La suma de estos eventos dio lugar a un escenario espacialmente complejo en cuanto a la disponibilidad de esta roca (Stern, 2004; Kay, Burns, Copeland y Mancilla, 2006; Andersen et al., 2017).

En particular, en el norte del Neuquén, dentro de la provincia geológica denominada Cuenca Neuquina, la formación de obsidiana se limita al magmatismo que se desarrolló durante el período Terciario. Las investigaciones arqueológicas que se desarrollan entre los 36 y $37^{\circ}$ de latitud sur y los 69 y $71^{\circ}$ de longitud oeste evidencian los siguientes tipos químicos de obsidiana: Cerro Huenul, Laguna del Maule 1-Laguna Negra y Laguna del Maule 2-Río Barrancas (Seelenfreund et al., 1996; Durán et al., 2004; Giesso et al., 2011; Cortegoso, Neme, Giesso, Durán y Gil, 2012; Barberena et al., 2018). En trabajos recientes se ha buscado profundizar en el conocimiento de estas fuentes a partir de una combinación de muestreos geoarqueológicos y de una sólida y abundante caracterización geoquímica de fuentes y artefactos (Fernández et al., 2017; Barberena et al., 2018). Además de complejizar el conocimiento disponible sobre las fuentes ya conocidas, estos trabajos han arrojado datos novedosos sobre tipos de obsidiana previamente desconocidos.

Se presenta aquí el registro más destacable de estas nuevas fuentes de obsidiana ubicado en el noroeste de la provincia de Neuquén, y denominado Varvarco por su ubicación en la cuenca fluvial homónima (Fig. 1). Estos resultados se integran a los obtenidos en los últimos años de investigación en la región que persiguieron reconstruir la estructura espacial de las fuentes de obsidiana, patrones de organización tecnológica, de movilidad y de interacción social medida mediante las propiedades del arte rupestre en este ámbito cordillerano altitudinal y ambientalmente heterogéneo (Barberena et al., 2017, 2018; Fernández et al., 2017).

\section{La obsidiana Varvarco en su contexto geográfico, ambiental, geológico y geomorfológico}

El área de estudio de este trabajo se ubica en el Dpto. de Minas, en el ángulo septentrional de la provincia del Neuquén $\left(36^{\circ} 15^{\prime} \mathrm{S}, 36^{\circ} 30^{\prime} \mathrm{O}\right)$, un paisaje caracterizado por la presencia de múltiples aparatos volcánicos del Neógeno-Cuaternario (González Díaz, Folguera, Costa, Wright y Ellisondo, 2006). El límite de este espacio lo conforman la Cordillera de los Andes al oeste y la serranía de Colomichi Có, primero, y la Cordillera del Viento, después, hacia el este. Entre estas cadenas montañosas se presentan planicies lávicas, serranías menores y valles fluviales. Los diferentes pisos

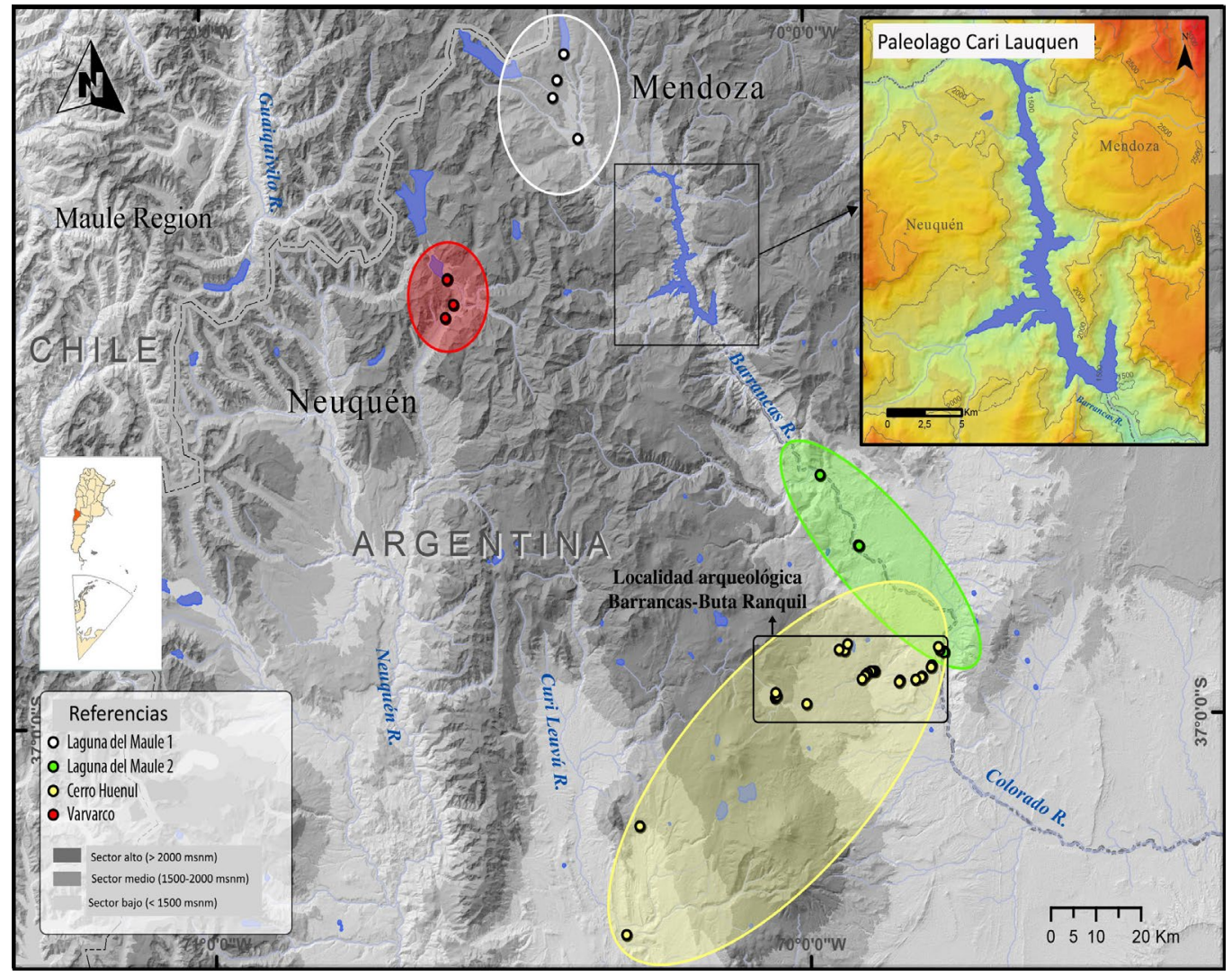
altitudinales crean condiciones para diferentes tipos de vegetación: desde desierto de altura hasta bosque, pasando por estepa herbácea y arbustiva. Un rasgo destacable son las vegas o mallines que constituyen recursos muy valorados en este ambiente árido (Movia, Ower y Pérez, 1982).

Figura 1. Área de estudio y ubicación de las principales fuentes de obsidiana y de la fuente Varvarco.

Figure 1. Study area and location of the main obsidian sources and Varvarco source. 


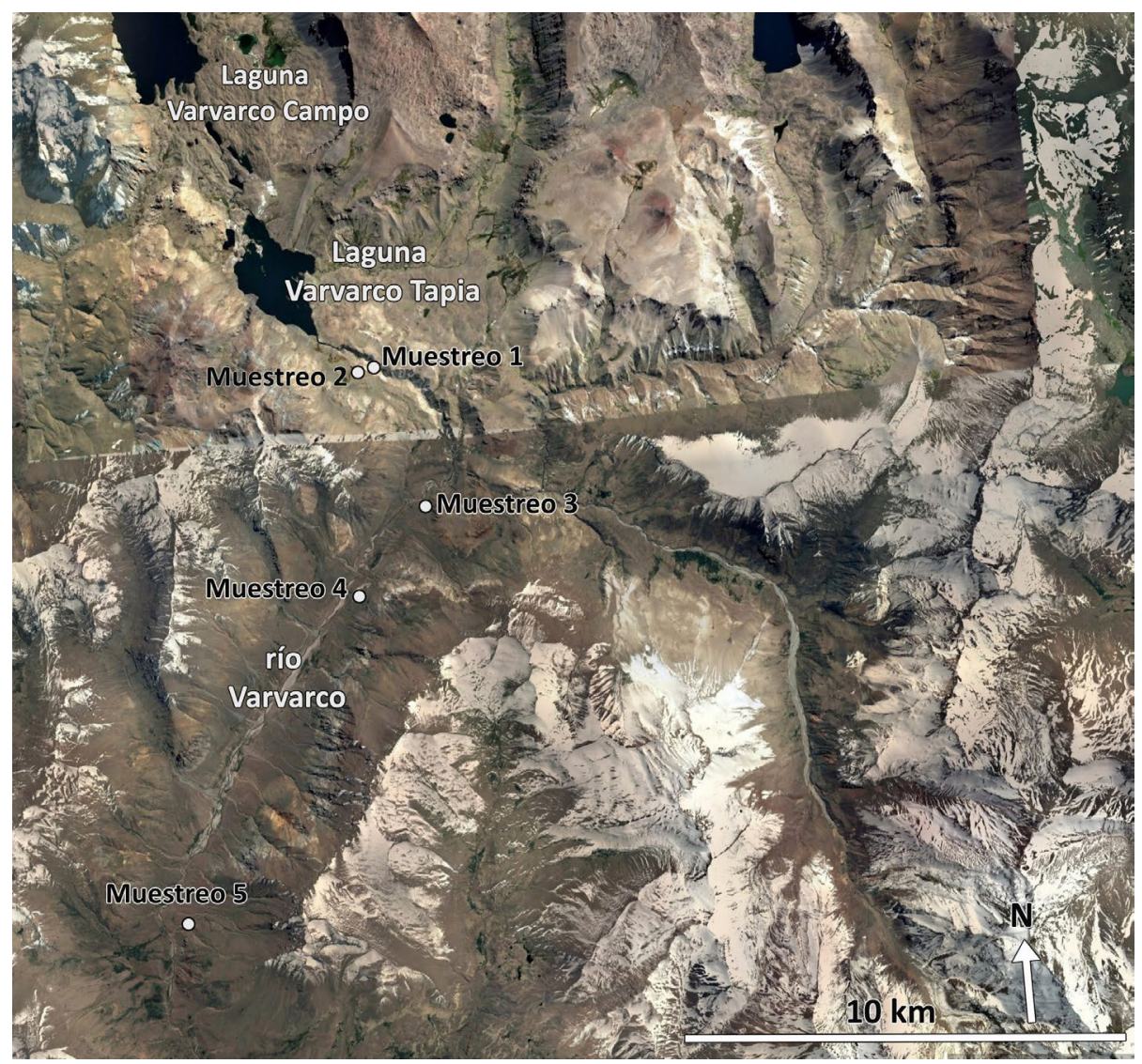

Figura 2. Detalle del nacimiento y tramo superior del río Varvarco y ubicación de los muestreos realizados.

Figure 2. Detail of the birth and upper section of the Varvarco river and location of the samplings carried out.

Las lagunas Varvarco Tapia y Varvarco Campos se ubican en el amplio valle labrado en el Pleistoceno por el Glaciar Troncal de Varvarco (González Díaz et al., 2000). La laguna Varvarco Campos es la más septentrional y desagua en la más pequeña que es la laguna Varvarco Tapia, cuyo desagüe da origen al río Varvarco (Fig. 2). Este presenta un fuerte control estructural y, en conjunto con el río Pichi Neuquén, son los dos afluentes principales del río Neuquén en su tramo inicial, con el cual confluye en una cota de 1140 m.s.n.m. (González Díaz et al., 2000; Beuret, 2014).

La columna estratigráfica del área de estudio comienza con rocas volcánicas del Pérmico Superior (Grupo Choiyoi) en las cuales, durante el Triásico Inferior, se intruyeron pequeños granitoides que se agrupan bajo la denominación Granito Varvarco Tapia. Luego de esta actividad magmática el área no experimentó actividad ígnea hasta el Eoceno Superior-Oligoceno, cuando se extruyen lavas andesíticas y basálticas con niveles de tobas y conglomerados (Fm. Cayanta). Durante el Mioceno y el Plioceno la zona experimentó un período de particular intensidad en la actividad magmática que se evidencia por la sucesión de coladas dacíticas (Fm. Turbio), seguidas de tobas y lavas andesíticas (Fm. Campanario), luego basaltos, aglomerados basálticos y tobas que componen a la Fm. Basalto Coyoco y finalmente coladas de andesitas (Fm. Andesita Tilhué) (Zanettini 2001). Durante el cuaternario la actividad magmática se desplazó hacia el este del área de estudio donde se extruyen lavas riolíticas de la Fm. Cerro Domo y traquitas, andesitas y traquiandesitas de la formación Andesitas Matru.

Los procesos exógenos modelaron el relieve hasta su forma actual, labrando valles desde las cotas mas elevadas y rellenando los bajos topográficos. Entre ellos predomina la impronta de la actividad glaciaria, seguida de la actividad fluvial y los procesos gravitacionales (González Díaz et al. 2000, 2003; Costa y Díaz 2007). En particular, las lagunas Varvarco Tapia y Varvarco Campos están asociadas con extensas avalanchas de rocas que se consideran parte responsable de su origen. La avalancha de la laguna Varvarco Campos ha sido reconocida como la de mayor extensión de la Argentina (Costa y González Díaz 2007). Estas avalanchas fueron fechadas mediante el isótopo ${ }^{36} \mathrm{Cl}$ y dieron fechas correspondientes al Pleistoceno final (Costa y González Díaz 2007).

\section{Materiales y métodos}

Prospección y muestreo de fuentes de obsidiana Los muestreos a lo largo del valle del río Varvarco han sido ubicados en diferentes pisos altitudinales, desde su origen, a ca. 2000 m.s.n.m., hasta su desembocadura en el río Neuquén, a ca. 1200 m.s.n.m. Partiendo del registro incidental de obsidiana en la cuenca media del río Varvarco, se diseñó una estrategia dirigida de muestreo geoarqueológico enfocada en dos objetivos: a) evaluar la procedencia de esta obsidiana transportada por el río, para lo cual se prospectó río arriba del hallazgo inicial, 
hasta las nacientes de la cuenca; y b) caracterizar la amplitud de la dispersión fluvial de la obsidiana, para lo cual se prospectó río abajo del área de hallazgo original. Esto se realizó en dos campañas de trabajo de campo desarrolladas en 2017 y 2018.

Se realizaron cinco muestreos en puntos distribuidos a lo largo de 18 km de la cuenca del río Varvarco (Fig. 2). En cada uno de ellos, cinco personas exploraron en forma intensiva un segmento de la cuenca de ca. $300 \mathrm{~m}$ lineales, abarcando tanto los espacios inmediatamente adyacentes al cauce actual, como sectores más alejados lateralmente. En cada contexto, se registraron y recuperaron nódulos de obsidiana representativos de la variación en tamaño y morfología observada.

En cada unidad de muestreo se registraron variables generales del paisaje tales como el tipo de geoforma, la pendiente, el relieve, la altitud, el tipo de sustrato y la cubierta vegetal. Además, se consignaron las siguientes características particulares para los depósitos de obsidiana: tipo de yacimiento (primario o secundario), forma de presentación (afloramiento, filón, venilla,
Inicialmente se analizaron todas las muestras mediante XRF. Luego, aquellas muestras de fuentes que no podían diferenciarse estadísticamente entre sí por la concentración de los elementos medidos por XRF, fueron sometidas a NAA. Los análisis de XRF fueron realizados usando un espectrómetro de energía dispersa "Thermo Fisher Scientific ARL Quant' $x$ ". Las muestras fueron analizadas durante dos minutos cada una, lo cual permitió medir los siguientes elementos: manganeso $(\mathrm{Mn})$, hierro $(\mathrm{Fe})$, zinc $(\mathrm{Zn})$, rubidio $(\mathrm{Rb})$, estroncio $(\mathrm{Sr})$, iridio $(\mathrm{Y})$, zircón (Z), niobio ( $\mathrm{Nb})$ y torio (Th). El espectrómetro utilizado para medir XRF fue calibrado para realizar los análisis de obsidiana a partir de la medición de 40 muestras de fuentes bien caracterizadas previamente analizadas por NAA, ICP-MS y XRF (ver Glascock y Ferguson, 2012).

\section{Resultados}

Prospección geoarqueológica y propiedades morfométricas de los nódulos

Se prospectaron y muestrearon de forma dirigida cinco sectores de la cuenca (Fig. 2). Los Puntos 1 y 2 de muestreo se ubican inmediatamente al sur de la laguna bloque, guijarro, clasto), forma de distribución (dispersa o concentrada) y abundancia (escasa, relativamente abundante o abundante).

Cada obsidiana recolectada fue analizada durante las tareas de campo y algunos ejemplares fueron seleccionados para trasladar al laboratorio para posteriores análisis. Las variables registradas para cada nódulo fueron: color, calidad para la talla (excelente, muy buena, buena, regular o mala), eje mayor (largo), eje intermedio (ancho), eje menor (espesor), redondeamiento (según diagrama de la Kent State University: $0.1,0.3,0.5,0.7$ y 0.9 , siendo 0.1 la categoría más angulosa y 0.9 la más redondeada), textura superficial (lisa, burbujas, estrías, incisiones o rugosa) y homogeneidad (homogéneo, inclusiones minerales, fracturas inherentes, venillas o múltiples cualidades indeseadas).

\section{Métodos de caracterización} geoquímica de las obsidianas Las muestras de obsidiana fueron analizadas usando una combinación de XRF y NAA. Se siguieron los procedimientos estándar del Archaeometry Laboratory perteneciente al Missouri University Research Reactor (EE.UU.).

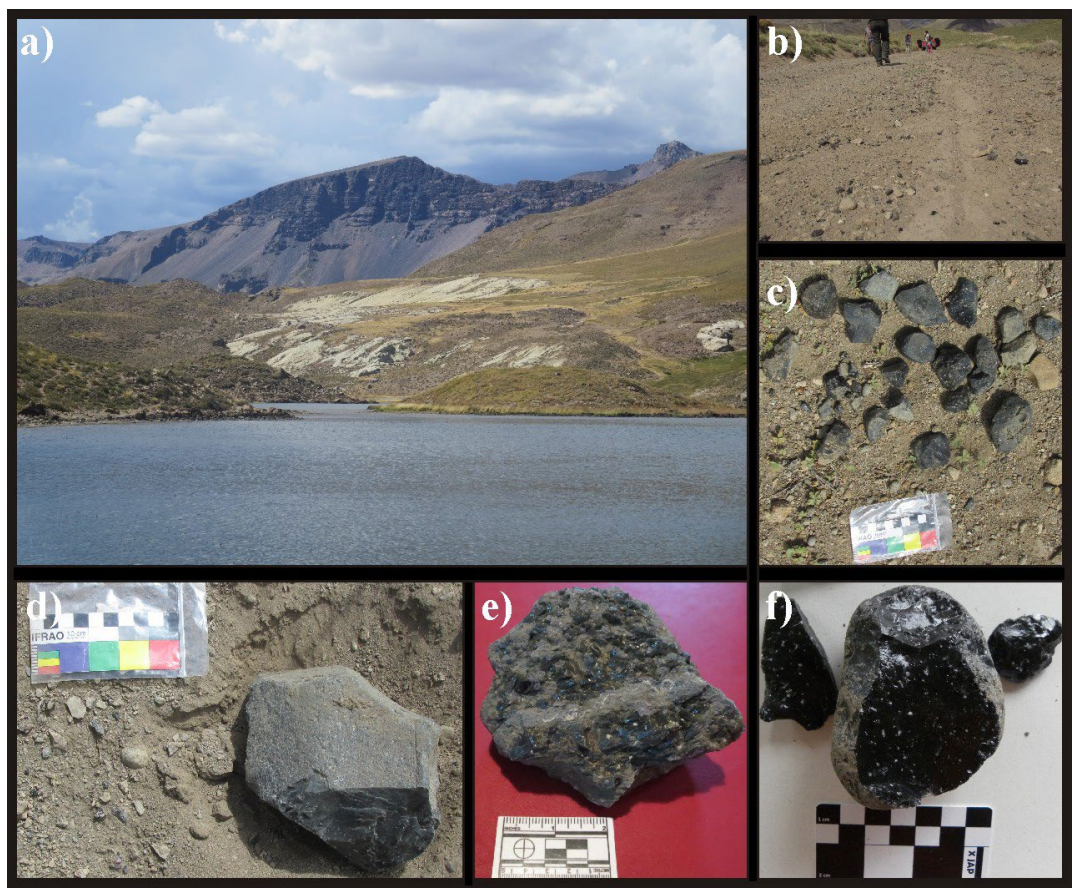

Figura 3. a) Laguna Varvarco Tapia, donde nace el río Varvarco y se ubican los puntos de muestreo 1 y 2 ; b) distribución de los nódulos de obsidiana en el terreno en el punto 1 de muestreo; c) recolección de nódulos de obsidiana en el punto 1 de muestreo; d) detalle de nódulo de obsidiana anguloso recolectado en el punto 2 de muestreo; e) obsidiana como inclusiones o venillas en un nódulo recolectado en el punto 1 de muestreo; y f) nódulo de obsidiana recolectado en el punto 4 de muestreo.

Figure 3. a) Varvarco Tapia lagoon, where the Varvraco river is born and where sampling points 1 and 2 are located; b) distribution of obsidian nodules in the field at sampling point 1 ; c) collection of obsidian nodules at sampling point 1 ; d) detail of an angled obsidian nodule collected at sampling point 2; e) obsidian as inclusions or veins in a nodule collected in sampling point 1; and f) obsidian nodule collected at sampling point 4. 
Varvarco Tapia, a ca. 2000 m.s.n.m. (Fig. 3a, b, c y d). Los Puntos 3 y 4 se localizan en el tramo medio, mientras que el Punto 5 se emplaza en el tramo inferior del río Varvarco. En los Puntos 1 a 4 de muestreo se detectó la presencia de obsidiana, mientras que en el 5 no fue registrada.

En los Puntos 1 y 2 de muestreo la obsidiana se encuentra asociada a amplios depósitos ignimbríticos que afloran en la superficie del terreno y son disectados por el río. Se ha muestreado sobre la terraza glacifluvial (Punto 1) y en la planicie aluvial actual (Punto 2). Se recolectaron 30 nódulos en el primer Punto y 12 en el segundo. Las obsidianas allí aparecen en depósitos secundarios y en algunos bloques no transportables y en nódulos transportables en forma de inclusiones o venillas (Fig. 3e). Los ejemplares se encuentran dispersos y son relativamente abundantes (Fig. 3b). Hemos registrado algunos que alcanzan tamaños mayores a los $300 \mathrm{~mm}$ en su eje mayor aunque la mayoría de los nódulos promedia en el Punto 1 apenas los $48 \pm 10 \mathrm{~mm}$ de largo y en el Punto 2 los $50 \pm 24 \mathrm{~mm}$. No se han registrado diferencias entre los ejemplares de estos dos puntos. La obsidiana es negra y de buena calidad para tallar, aunque presenta fracturas inherentes y cristaloclastos. Los nódulos son mayormente angulosos y la textura superficial es lisa (Fig. 3d). Hemos registrado algunos pocos ejemplares bandeados. En el Punto 1 hemos registrado artefactos, que demuestran que la obsidiana ha sido aprovechada efectivamente como materia prima, confirmando su carácter de fuente de aprovisionamiento en el pasado.

El Punto 3 se localiza casi ocho kilómetros más al sur, en la planicie aluvial actual del río Varvarco, en un punto donde un puente cruza el río a ca. 1700 m.s.n.m. Alí recolectamos 13 nódulos en forma selectiva. Las obsidianas aparecen en depósitos secundarios de origen glacifluvial y de erosión lateral del valle. Los nódulos aquí aparecen dispersos y son escasos. Son negros y su calidad para la talla es buena aunque, como en el caso anterior, la mayoría registra fracturas inherentes e inclusiones minerales. En este punto de muestreo los tamaños promedio son mayores que en los anteriores $(61 \pm 48 \mathrm{~mm}$ en su eje mayor), los nódulos son en general redondeados y su textura superficial es lisa. Sólo un ejemplar exhibe bandeamiento.

El Punto 4 se encuentra en la planicie aluvial subactual del río Varvarco, 2,5 km al sur del Punto 3 (Fig. 3) a una altitud de 1715 m.s.n.m (corresponde al emplazamiento del hallazgo original). Aquí registramos 34 nódulos de obsidiana disponibles en forma secundaria, dispersa y relativamente abundante. Son de color negro, aunque algunas son grises. Su calidad para tallar es buena; sin embargo, la mayoría presenta fracturas y cristaloclastos (Fig. 3f). El tamaño de los ejemplares en promedio es de casi $39 \pm 22 \mathrm{~mm}$ en su eje mayor, es decir que son más pequeños que los registrados en los Puntos 1, 2 y 3, lo cual es consistente con su posición en la cuenca. La textura superficial exhibe incisiones en la mayoría de los casos y el redondeamiento es intermedio.

El Punto 5 corresponde a un muestreo realizado sobre la planicie actual del río Varvarco a una altitud de ca. 1600 m.s.n.m. Los depósitos muestreados corresponden a acumulaciones glacifluviales mezcladas con depósitos muy sueltos producto del desprendimiento de materiales de las laderas del valle por derrumbes transportados por cárcavas, canales y escurrideros. A pesar de tratarse de una localización ubicada a sólo $12 \mathrm{~km}$ del Punto 4, y de la inspección exhaustiva de un amplio segmento de la cuenca, no registramos obsidiana.

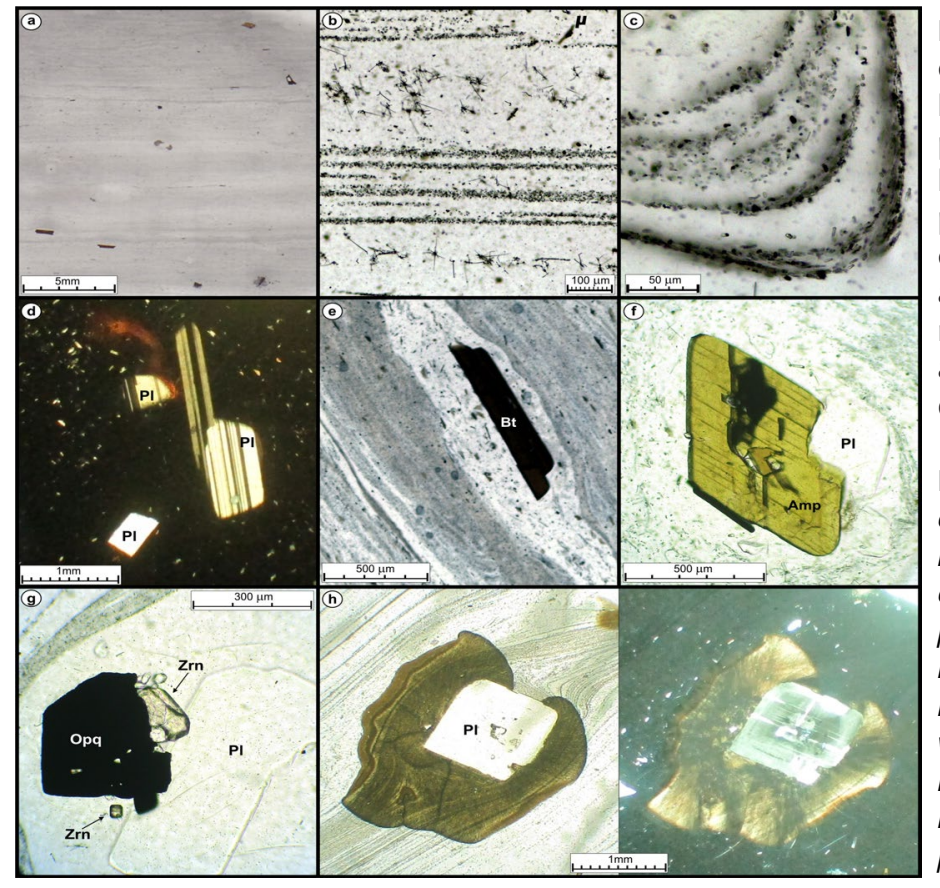

Figura 4. a) bandeamiento subparalelo típico de las obsidianas Varvarco; b) detalle con nicoles paralelos del bandeamiento más frecuente; c) microlitos de minerales prismáticos incoloros y minerales opacos orientados según el bandeamiento; d) cristaloclastos de plagioclasa con maclas polisintéticas (nicoles cruzados); e) cristaloclasto de biotita orientado según el bandeamiento; f) cristaloclasto típico de anfíbol junto a plagioclasa y opacos; g) cristaloclastos de mineral opaco junto a plagioclasa y prismas de circones; $h$ ) alteración incipiente del vidrio alrededor de un cristaloclasto de plagioclasa (sin y con nicoles cruzados).

Figure 4. a) Subparallel banding typical of Varvarco obsidians; b) detail with parallel nicols of the most frequent banding; c) microliths of colorless prismatic minerals and opaque minerals oriented according to the banding; d) plagioclase crystalloclast with polysynthetic twins (crossed nicols); e) biotite crystalloclaste oriented according to the banding; f) crystalloclast typical of amphibole together with plagioclase and opaque; g) crystalloclast of opaque mineral together with plagioclase and zircon prisms; h) incipient alteration of the glass around a crystalloclast of plagioclase (without and with crossed nicols). 


\section{Petrografía de la obsidiana Varvarco}

Para la caracterización petrográfica de la obsidiana se seleccionaron seis muestras que representan a los tipos macroscópicamente distintivos y fueron extraídas del punto 4 de muestreo (Fig. 1). La obsidiana Varvarco está formada por la mezcla de al menos dos vidrios cuya proporción relativa provoca diferentes tonalidades macroscópicas y texturas bandeadas finas que a veces sólo se aprecia a escala microscópica (Fig. 4a). Dicho bandeamiento resulta de la alternancia de bandas castañas y bandas incoloras con espesores que varían de 1 a 0,15 mm (Fig. 4a). En menor proporción, otros ejemplares poseen texturas crenuladas producto de deformación sobreimpuesta. El porcentaje relativo de cada uno de los vidrios varía significativamente desde muestras con proporciones iguales hasta otras compuestas sólo de vidrio incoloro. En conjunto, el vidrio representa más del $80 \%$ del volumen total de las muestras estudiadas.

El vidrio incoloro es el más abundante y destaca por su estado fresco de alteración y su elevada concentración de microlitos cristalizados durante la extrusión (Fig. 4a). Entre los microlitos que así se formaron predominan prismas cortos de tamaños inferiores a los $10 \mu \mathrm{m}$ que se orientan en forma subparalela al bandeamiento (Fig 4b, c).

El restante $20 \%$ de la muestra se compone de cristaloclastos incorporados mecánicamente durante la extrusión puesto que las bandas y los microlitos antes descriptos los circundan copiando la forma de sus contornos (Fig. 4e, h). Los más abundantes son prismas de plagioclasas de un milímetro de largo promedio (Fig. 4d) que evidencian una incipiente desvitrificación muy restringida del vidrio que las rodea (Fig. 4h). En menor proporción le siguen láminas de biotita sin evidencias de alteración ni deformación

Tabla 1. Concentraciones de elementos de las muestras de obsidiana Varvarco medidas por XRF.

Table 1. Elements concentrations of Varvarco obsidian samples mesure by XRF.

\begin{tabular}{lccccccccccc}
\hline Muestra & Grupo & Gn & Fe & Zn & Rb & Sr & Y & Zr & Nb & Th \\
& Geoquímico & & & & & & & & & \\
\hline RAM051 & Varvarco & 447,5 & 7456,6 & 22,0 & 188,4 & 98,0 & 20,0 & 148,5 & 13,1 & 29,4 \\
RAM052 & Varvarco & 435,1 & 8671,3 & 24,2 & 190,2 & 99,6 & 19,0 & 146,1 & 14,2 & 29,2 \\
RAM053 & Varvarco & 447,1 & 7417,2 & 24,8 & 190,5 & 89,8 & 18,8 & 142,6 & 14,0 & 28,2 \\
RAM054 & Varvarco & 486,7 & 8409,5 & 27,8 & 198,3 & 101,5 & 19,1 & 152,0 & 13,0 & 28,6 \\
RAM055 & Varvarco & 454,2 & 7420,6 & 21,7 & 190,1 & 93,1 & 18,7 & 154,4 & 12,8 & 27,8 \\
RAM056 & Varvarco & 450,5 & 8199,9 & 20,7 & 194,0 & 129,3 & 19,0 & 150,7 & 13,4 & 27,0 \\
RAM057 & Varvarco & 473,3 & 8163,9 & 27,9 & 198,0 & 98,4 & 18,4 & 150,5 & 12,6 & 29,5 \\
RAM058 & Varvarco & 476,0 & 8483,3 & 29,3 & 206,1 & 102,4 & 20,3 & 159,8 & 12,3 & 27,6 \\
RAM059 & Varvarco & 441,9 & 7763,3 & 27,3 & 182,6 & 89,4 & 17,8 & 140,1 & 11,3 & 24,6 \\
RAM060 & Varvarco & 521,9 & 9410,9 & 31,4 & 207,3 & 146,6 & 19,1 & 151,6 & 13,4 & 29,3 \\
RAM061 & Varvarco & 432,2 & 7030,4 & 23,0 & 179,7 & 92,9 & 18,1 & 139,0 & 12,5 & 24,5 \\
\hline
\end{tabular}

mecánica y cuyo tamaño no supera los 0,5 mm (Fig. 4e). Completan la fracción de cristaloclastos prismas de anfíboles de tamaños inferiores a 0,5 mm en estado fresco de alteración (Fig. 4f). Por último, se encuentran en cantidades accesorias (>1\%) minerales opacos y prismas de circones (Fig 4g).

Es importante destacar la homogeneidad de las muestras en las características del vidrio y en la composición de los cristaloclastos. La diferencia entre las seis muestras observadas radica fundamentalmente en la cantidad de vidrio castaño. Esta similitud petrográfica de las muestras sugiere que todas han experimentado los mismos procesos geológicos y, por lo tanto, sustenta la hipótesis de una misma fuente primaria para todas ellas. Por otro lado, el estado fresco del vidrio y la baja cantidad de cristaloclastos permiten asegurar que la impronta geoquímica de las muestras refleja la composición de la obsidiana primaria.

\section{Geoquímica de la obsidiana Varvarco}

Del total de 89 nódulos recolectados en todos los muestreos, se seleccionaron once muestras para analizar por XRF (Tabla 1). De éstas, nueve fueron medidas también por NAA (Tabla 2). La totalidad de las muestras provienen del punto 4 de muestreo dado que era el único muestreo realizado al momento de hacer los análisis, pero los mismos se ampliarán a los muestreos restantes.

Para definir a la obsidiana Varvarco como un tipo geoquímico distinto a los ya conocidos utilizamos el postulado de proveniencia, que sostiene que la variabilidad elemental, isotópica y/o mineralógica entre fuentes o grupos geoquímicos debe ser mayor a la variabilidad dentro de un mismo grupo composicional

(Weigand, Harbottle y Sayre, 1977; Glascock y Neff, 2003). Así, vemos que las muestras de obsidiana Varvarco se diferencian de las obsidianas Cerro Huenul, Fuente Alba, Laguna del Maule 1-Laguna Negra y Laguna del Maule 2-Río Barrancas, conformando un nuevo grupo geoquímico para el noroeste de la Patagonia (Fig. 5).

En la Figura 5b se observa que, mientras que la información bivariada obtenida para Hf y Cs mediante NAA discrimina en forma consistente al tipo químico Varvarco de los demás reconocidos a nivel regional, los resultados obtenidos mediante XRF muestran un solapamiento considerable en el rango de valores, particularmente con el tipo químico Laguna del Maule 2-Río Barrancas (Fig. 5a). Dado que el método de NAA es no sólo más costoso, sino también destructivo y no puede ser realizado mediante equipos portátiles, resulta prioritario profundizar la capacidad discrminatoria asequible con los datos de XRF a fin de poder desarrollar una agenda eficiente 


\begin{tabular}{lcccccccccccccccc}
\hline Muestra & $\begin{array}{c}\text { Grupo } \\
\text { Geoquímico }\end{array}$ & $\mathrm{La}$ & $\mathrm{Lu}$ & $\mathrm{Nd}$ & $\mathrm{Sm}$ & $\mathrm{U}$ & $\mathrm{Yb}$ & $\mathrm{Ce}$ & $\mathrm{Co}$ & $\mathrm{Cs}$ & $\mathrm{Eu}$ & $\mathrm{Fe}$ & $\mathrm{Hf}$ & $\mathrm{Rb}$ & $\mathrm{Sb}$ & $\mathrm{Sc}$ \\
\hline RAM051 & Varvarco & 33,7 & 0,3 & 21,3 & 4,1 & 6,7 & 2,1 & 63,7 & 0,6 & 8,3 & 0,5 & 7973,1 & 4,9 & 185,3 & 0,4 & 2,0 \\
RAM052 & Varvarco & 34,0 & 0,3 & 20,9 & 4,1 & 6,0 & 2,1 & 64,8 & 0,6 & 8,4 & 0,5 & 8042,4 & 4,8 & 188,4 & 0,4 & 2,1 \\
RAM055 & Varvarco & 33,2 & 0,3 & 20,1 & 4,1 & 6,3 & 2,0 & 63,4 & 0,6 & 8,3 & 0,5 & 8054,3 & 4,6 & 185,7 & 0,4 & 2,1 \\
RAM056 & Varvarco & 34,2 & 0,3 & 21,5 & 4,2 & 6,2 & 2,1 & 65,9 & 0,8 & 8,5 & 0,5 & 8385,7 & 4,8 & 191,2 & 0,4 & 2,1 \\
RAM057 & Varvarco & 33,1 & 0,3 & 19,7 & 4,1 & 6,4 & 2,0 & 63,8 & 0,8 & 8,3 & 0,5 & 8031,8 & 4,7 & 184,8 & 0,4 & 2,1 \\
RAM058 & Varvarco & 33,1 & 0,3 & 21,8 & 4,1 & 6,1 & 2,0 & 64,2 & 0,7 & 8,4 & 0,5 & 8018,5 & 4,8 & 187,1 & 0,4 & 2,1 \\
\multirow{2}{*}{ RAM059 } & Varvarco & 32,9 & 0,3 & 21,0 & 4,1 & 6,3 & 2,0 & 64,8 & 0,6 & 8,4 & 0,5 & 8281,5 & 4,9 & 189,1 & 0,4 & 2,1 \\
RAM060 & Varvarco & 33,0 & 0,3 & 20,1 & 4,0 & 6,2 & 2,1 & 62,9 & 0,8 & 8,2 & 0,5 & 8123,6 & 4,7 & 184,4 & 0,4 & 2,1 \\
RAM061 & Varvarco & 33,6 & 0,3 & 20,8 & 4,2 & 6,2 & 2,1 & 65,8 & 0,8 & 8,5 & 0,5 & 8750,6 & 5,4 & 192,1 & 0,4 & 2,3 \\
\hline
\end{tabular}

Tabla 2. Concentraciones de elementos de las muestras de obsidiana Varvarco medidas por NAA.

Table 2. Elements concetrations of Varvarco obsidian samples mesure by NAA.

de investigación geoquímica en esta nueva región. Con este objetivo, se han explorado las proporciones entre distintos pares de elementos químicos (Fig. 6).

Estudios previos han demostrado que múltiples fuentes de obsidiana que presentan perfiles geoquímicos similares pueden ser discriminadas en forma más fehaciente mediante proporciones entre elementos químicos que mediante los valores de concentración en forma aislada (Glascock, Braswell y Cobean, 1998). Para los datos presentados aquí se exploraron todas las combinaciones posibles entre los nueve elementos químicos caracterizados mediante XRF ( $M n, F e, Z n, R b, S r$, $\mathrm{Y}, \mathrm{Zr}, \mathrm{Nb}, \mathrm{Th})$, que es el tipo de datos cuya resolución se quiere maximizar. En la Figura 6 se presentan las relaciones entre $\mathrm{Zr} / \mathrm{Th}$ versus $\mathrm{Rb} / \mathrm{Mn}$, and $\mathrm{Zr} / \mathrm{Rb}$ versus $\mathrm{Mn}$ /Fe para los tres tipos químicos que resultan más semejantes entre sí: Varvarco, Laguna del Maule 1 y Laguna del Maule 2. Allí puede observarse que estos indicadores diferencian en forma más robusta a estas fuentes que los datos originales de concentración elemental. Esta observación es clave de cara al trabajo futuro de asignación química de artefactos mediante XRF en el noroeste de Patagonia, que resulta la tecnología más eficiente en términos de costos, tiempo y carácter no destructivo en comparación con NAA. No obstante, será muy importante validar la eficacia de estas relaciones químicas mediante amplias bases de datos para artefactos de la región.

\section{Discusión y conclusiones}

Formación y distribución de los depósitos de obsidiana en el paisaje

Sintetizando la información presentada, se cuenta con un registro que indica que el punto de depositación original de las obsidianas mediante procesos piroclásticos ubica la fuente en las márgenes de la laguna Varvaco Tapia. Allí registramos depósitos ignimbríticos compuestos por bloques de diámetros que superan 1,5 m que incluyen fragmentos de obsidiana. Dada su posición estratigráfica estos depósitos pueden correlacionarse por el momento con la formación Andesitas Matru puesto que sus niveles tobáceos ricos en obsidianas representan al único evento magmático del área que registra la cristalización de estos productos volcánicos (Narciso, Santamaría y Zanettini, 2000; Danieli et al., 2011). Sin embargo,
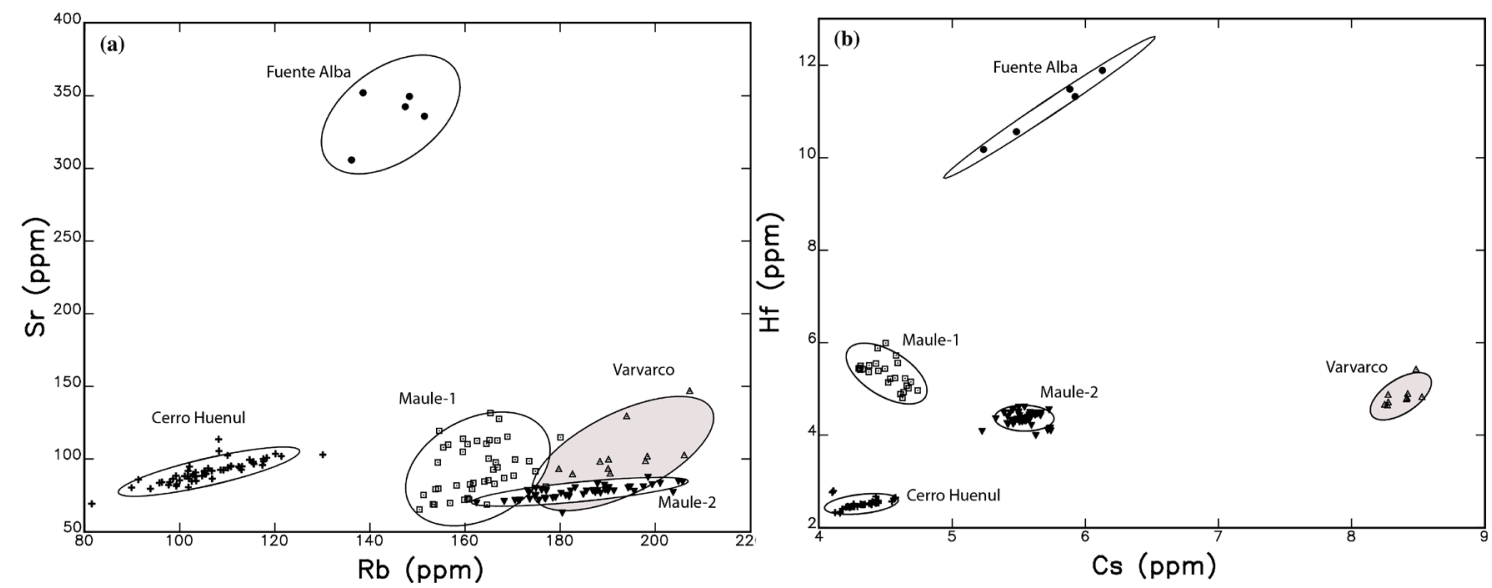

Figura 5. a) Correlación de rubidio versus estroncio medidos por XRF de las obsidianas de Neuquén; b) Correlación de hafnio versus cesio medidos por NAA para las mismas fuentes (las elipses representan un intervalo de confianza del 90\%).

Figure 5. a) Scatterplot of rubidium versus strontium XRF-generated data for Neuquén obsidian sources; b) scatterplot of cesium versus hafnium NAA-generated data for the same sources (ellipses are drawn at 90\% confidence). 

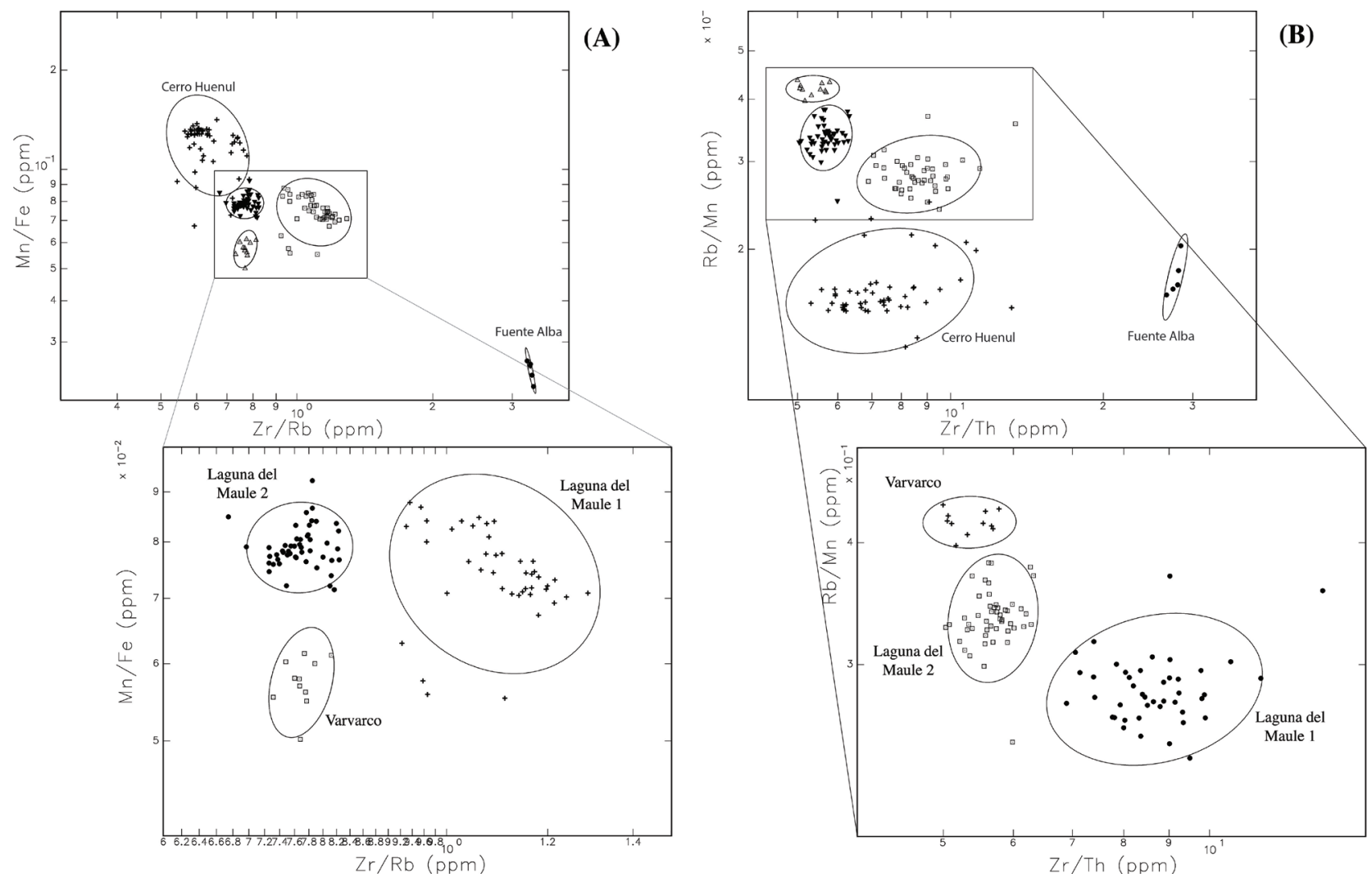

Figura 6. a) Correlación de $\mathrm{Zr} / \mathrm{Th}$ versus Rb/Mn de los datos generados por XRF para las fuentes de Varvarco, Cerro Huenul, Fuente Alba, Laguna del Maule 1, y Laguna del Maule2; b) Separación de las fuentes Varvarco, Laguna del Maule 1 y Laguna del Maule 2 (las elipses representan un intervalo de confianza del 90\%).

Figure 6. a) Scatterplot of Zr/Th versus Rb/Mn XRF data showing distribution of sources Varcarco, Cerro Huenul, Fuente Alba, Maule-1, and Maule-2; b) inset showing separation of Varvarco, Maule-1 and Maule-2 sources (all ellipses are drawn at $90 \%$ confidence).

otra posibilidad es que las obsidianas se asocien con la formación Tilhué, que aflora en cercanías del área, tiene una amplia extensión regional, aunque discontinua, y sabemos efectivamente que incluye manifestaciones de obsidiana en otros puntos del norte de Neuquén, siempre coincidentes con el tipo químico Cerro Huenul (Barberena et al., 2011). Independientemente de este aspecto geológico, hemos podido confirmar también la dispersión fluvial de esta obsidiana hacia el suroeste por el río Varvarco. Aunque no puede descartarse aún la presencia de esta obsidiana hacia el sur de los contextos fluviales que hemos muestreado, los datos actualmente disponibles confirman el transporte fluvial de obsidiana hasta unos $10 \mathrm{~km}$ de distancia de las ignimbritas que constituyen el emplazamiento primario. El Punto 5 de muestreo, ubicado a $19 \mathrm{~km}$ de dicha zona original de depositación, no presenta obsidiana. De confirmarse este registro, se trata entonces de una fuente que abarca ignimbritas en el contexto perilacustre y depósitos fluviales de la cuenca superior del río Varvarco. Una mejor definición del límite en la dispersión natural de esta obsidiana será importante para la realización de interpretaciones espaciales a partir del registro arqueológico.

El significado arqueológico de la fuente de obsidiana

\section{Varvarco}

Aunque la obsidiana Varvarco no presenta características óptimas para las actividades de talla lítica, ofrece una aptitud adecuada y nódulos de tamaños grandes que permiten la confección de todo tipo de instrumentos. De hecho, en nuestros muestreos iniciales hemos registrado contextos arqueológicos que contienen esta obsidiana bajo la forma de artefactos. Desde un punto de vista geográfico, el emplazamiento de esta fuente es accesible en forma anual y se localiza en la cuenca del río Neuquén, que es un espacio clave en escala macro-regional en términos de su elevada capacidad de carga de herbívoros y aptitud para la circulación humana (Barberena, 2013). A nivel macroregional, esta cuenca concentra tanto las mayores densidades de arte rupestre (Fernández, 2000[1979]; Cúneo, 2017), como de entierros humanos (Della Negra, 2008; Bernal, Cobos, Pérez y González, 2017), lo cual la señala como un espacio de alta jerarquía en los circuitos de movilidad y asentamiento.

La información aquí aportada sobre las propiedades geográficas, geológicas y geoquímicas de la nueva fuente de obsidiana Varvarco se suma al cuerpo de datos que se está generando a nivel macroregional para fuentes en espacios de altura y tierras bajas del norte de Patagonia y 
Cuyo (Durán et al., 2004; Giesso et al., 2011; Cortegoso et al., 2012). La identificación de este nuevo tipo químico ofrece un marcador geográfico para la cuenca superior del río Neuquén, que resulta un corredor de circulación y asentamiento anual clave en la escala del noroeste de Patagonia.

San Carlos de Bariloche, 26 de Octubre de 2018.

\section{Agradecimientos}

Esta investigación es financiada por el CONICET (PIP 0301) y la Agencia Nacional de Promoción de la Ciencia y la Tecnología de Argentina (PICT 2014-0940 y 201-0062). El Missouri University Research Reactor ha recibido apoyo mediante un subsidio de National Science Foundation (Grant 1621158). Mónica Berón y Martín Giesso ofrecieron comentarios y nos permitieron citar su trabajo inédito. Agradecemos a la Subsecretaría de Cultura de la Provincia del Neuquén y a los miembros de nuestro equipo por su ayuda permanente.

\section{Bibliografía}

Andersen, N.L., Singer, B.S., Jicha, B.R., Beard, B.L., Johnson, C.M. y Licciardi, J.M. 2017. Pleistocene to Holocene Growth of a Large Upper Crustal Rhyolitic Magma Reservoir beneath the Active Laguna del Maule Volcanic Field, Central Chile. Journal of Petrology 58(1): 85-114.

Barberena, R. 2013. Biogeografía, competencia y demarcación simbólica del espacio: modelo arqueológico para el norte de Neuquén. Intersecciones en Antropología 14: 367-382.

Barberena, R., M.V. Fernández, A.A. Rughini, K. Borrazzo, R. Garvey, G. Lucero, C. Della Negra, G. Romero V., V. Durán, V. Cortegoso, M. Giesso, C. Klesner, B.L. MacDonald y M.D. Glascock. 2018. Deconstructing a Complex Obsidian 'Source-scape': A Geoarchaeological and Geochemical Approach in Northwestern Patagonia. Geoarchaeolog, 2018: 1-12. https://doi.org/10.1002/ gea.21701.

Barberena, R., A. Hajduk, A. Gil, G. Neme, V. Durán, M.D. Glascock, M. Giesso, K. Borrazzo, M.P. Pompei, M.L. Salgán, V. Cortegoso, G. Villarosa y A. Rughini. 2011. Obsidian in the south-central Andes: geological, geochemical, and archaeological assessment of north Patagonian Sources (Argentina). Quaternary International 245 (1): 25-36.

Barberena, R., G. Romero Villanueva, G. Lucero, M.V. Fernández, A.A. Rughini y P. Sosa. 2017. Espacios internodales en Patagonia septentrional: biogeografía, información y mecanismos sociales de interacción. Estudios Atacameños 56: 57-75.
Bernal, V., V. Cobos, S.I. Perez y P.N. González. 2017. La estructura espacial del registro bioarqueológico de la provincia del Neuquén durante el Holoceno. En F. Gordón, R. Barberena y V. Bernal (Eds.), El poblamiento humano del norte del Neuquén: estado actual del conocimiento y perspectivas, pp. 123-144, Aspha, Buenos Aires.

Berón, M.A., F.N. Páez, M.P. Carrera Aizpitarte, M. Giesso y M.D. Glascock. 2018. Procurement and circulation of obsidian in the Province of La Pampa, Argentina. Ms en revisión.

Beuret, L.A. 2014. Estudio aprovechamiento multipropósito Colo-Michi Có y Los Guiones en la Alta cuenca del río Neuquén en la Provincia del Neuquén. Secretaría de Energía de la Nación. Licitación pública Internacional. Programa de Estudios en el Sector Energético en la República Argentina. Circular Aclaratoria $\mathrm{N}^{\circ} 2$.

Cortegoso, V, Neme, G., Giesso, M., Durán, V., Gil, A. (2012). El uso de la obsidiana en el sur de Mendoza. En G. Neme y A. Gil (Eds.), Paleoecología humana en el Sur de Mendoza, pp. 181-211, Sociedad Argentina de Antropología, Buenos Aires.

Costa, C.H. y E.F. González Díaz. 2007. Age constraints and paleoseismic implication of rock avalanches in the northern Patagonian Andes, Argentina. Journal of South American Earth Sciences 24: 48-57.

Cúneo, E.M. 2017. Sitio Buraleo: representaciones rupestres del "estilo Guaiquivilo" en el noroeste neuquino, departamento Minas, República Argentina. En F. Gordón, R. Barberena y V. Bernal (Eds.), El poblamiento humano del norte del Neuquén: estado actual del conocimiento y perspectivas, pp. 75-100, Aspha, Buenos Aires.

Danieli, J.C., O. Carbone, M. Franchini, A. Garrido, M. Gingins y H.A. Leanza. 2011. Sitios de Interes Geológico. Relatorio del XVIII Congreso Geológico Argentino: 881893.

Della Negra, C. 2008. Gubevi I: un sitio con restos óseos humanos asociados a cerámica en el Departamento Minas, Zona norte de la Provincia del Neuquén. En P. Azar, E. Cúneo y S. Rodríguez (Eds.), Tras la senda de los ancestros. Arqueología de Patagonia, EDUCO, Neuquén.

Durán, V., Giesso, M., Glascock, M.D., Neme, G., Gil, A., y Sanhueza, L. 2004. Estudio de fuentes de aprovisionamiento y redes de distribución de obsidiana durante el Holoceno Tardío en el sur de Mendoza (Argentina). Estudios Atacameños 28: 25-43.

Fernández, J. 2000 [1979]. Las piedras con marcas de la cordillera del Viento. Arte rupestre en el departamento 
Minas, Neuquén, Argentina. Sociedad Argentina de Antropología, Buenos Aires.

Fernández, M.V., R. Barberena, A. Rughini, M. Giesso, V.A. Durán, V. Cortegoso, K. Borrazzo, G. Lucero, G. Romero Villanueva, R. Garvey, B.L. MacDonald y M.D. Glascock. 2017. Obsidian geochemistry, geoarchaeology, and lithic technology in northern Patagonia (Argentina). Journal of Archaeological Science: Reports 13: 372-381.

Giesso, M., Durán, V., Neme, G., Glascock, M.D., Cortegoso, V., Gil, A., y Sanhueza, L. 2011. A study of obsidian source usage in the central Andes of Argentina and Chile. Archaeometry 53: 1-21.

Glascock, M.D., Braswell, G.E., y Cobean, R.H. 1998. A systematic approach to obsidian source characterization. En M.S. Shackley (Ed.), Archaeological Obsidian Studies: Method and Theory, pp. 15-65, Plenum Press, New York and London.

Glascock, M.D. y J.E. Ferguson. 2012. Report on the Analysis of Obsidian Source Samples by Multiple Analytical Methods. Archaeometry Lab, University of MissouriColumbia.

Glascock, M.D. y Neff, H. 2003. Neutron activation analysis and provenance research in archaeology. Measurement Science and Technology 14: 1516-1526.

González Díaz, E.F., L.E. Fauqué, A.D. Glaccardi y C.H. Costa. 2000. Las lagunas de Varvar co Campos y Varvra co Tapia ( $\mathrm{N}$ del Neuquén, Argentina): su relación con avalanchas de rocas. Revista de la Asociación Geológica Argentina, 55 (3): 147-164.

González Díaz, E.F., C.H. Costa y A.D. Giaccardi. 2003. El complejo deslizamiento de Ailinco-Cerro Papas-Las Olletas (Departamento Minas, norte del Neuquén, Argentina). Revista de la Asociación Geológica Argentina 58 (2): 194-200.
González Díaz, E.F., A. Folguera, C.H. Costa, E. Wright y M. Ellisondo. 2006. Los grandes deslizamientos de la región septentrional neuquina entre los $36^{\circ}-38^{\circ} \mathrm{S}$ : Una propuesta de inducción sísmica. Revista de la Asociación Geológica Argentina 61 (2): 197-217.

Kay, S., Burns, W., Copeland, P., \& Mancilla, O. 2006. Upper Cretaceous to Holocene magmatism and evidence for transient Miocene shallowing of the Andean subduction zone under the northern Neuquén Basin. En S. Kay \& V. Ramos (Eds.), Evolution of an Andean margin. A tectonic and magmatic view from the Andes to the

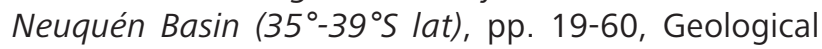
Society of America Special Paper 407, New York.

Movia, C., G.H. Ower y C.E. Pérez. 1982. Estudio de la vegetación natural de la Provincia del Neuquén. Ministerio de Economía y Hacienda, Secretaría de Recursos Naturales, Neuquén.

Narciso, V., Santamaría, G. y Zanettini, J.C. 2000. Hoja Geológica 3769-I, Barrancas. Escala 1: 250.000. Servicio Geológico Minero Argentino, Buenos Aires.

Ross C.S. 1962. Microlites in glassy volcanic rocks. The American Mineralogist 47: 723-740.

Seelenfreund, A., C. Rees, R. Bird, G. Bailey, R. Bárcena, y V. Durán. 1996. Trace element analysis of obsidian sources and artifacts of Central Chile (Maule River Basin) and Western Argentina (Colorado River). Latin American Antiquity 7: 7-20.

Weigand, P.C., G. Harbottle, y E. Sayre. 1977. Turquoise sources and source analysis: Mesoamerica and the southwestern USA. En T. Early \& J. Ericson (Eds.), Exchange Systems in Prehistory, pp. 15-32, Academic Press, New York.

Zanettini, J.J.C. 2001. Hoja Geológica Las Ovejas (3772II). Provincia de Neuquén. SEGEMAR, Buenos Aires. 